\title{
A NOTE ON THE SUPPORT NORM OF A CONTACT STRUCTURE
}

\author{
JOHN A. BALDWIN AND JOHN B. ETNYRE
}

\begin{abstract}
In this note we observe that the no two of the three invariants defined for contact structures in 3] - that is, the support genus, binding number and support norm - determine the third.
\end{abstract}

In [3], the second author and B. Ozbagci define three invariants of contact structures on closed, oriented 3-manifolds in terms of supporting open book decompositions. These invariants are the support genus, binding number and support norm. There are obvious relationships between these invariants, but [3] leaves open the question of whether any two of them determine the third. We show in this note that this is not the case.

Recall that an open book decomposition $(L, \pi)$ of a $3-$ manifold $M$ consists of an oriented link $L$ in $M$ and a fibration $\pi:(M-L) \rightarrow S^{1}$ of the complement of $L$ whose fibers are Seifert surfaces for $L$. The fibers $\pi^{-1}(\theta)$ of $\pi$ are called pages of the open book and $L$ is called the binding. It is often convenient to record an open book decomposition $(L, \pi)$ by a pair $(\Sigma, \phi)$, where $\Sigma$ is a compact surface which is homeomorphic to the closure of a page of $(L, \pi)$, and $\phi: \Sigma \rightarrow \Sigma$ is the monodromy of the fibration $\pi$. A contact structure $\xi$ on $M$ is said to be supported by the open book decomposition $(L, \pi)$ if $\xi$ is the kernel of a 1-form $\alpha$ which evaluates positively on tangent vectors to $L$ that agree with the orientation of $L$, and for which $d \alpha$ restricts to a positive volume form on each page of $(L, \pi)$.

With this in mind, we may describe the three invariants defined in [3]. The support genus of a contact structure $\xi$ on $M$ is defined to be

$$
\operatorname{sg}(\xi)=\min \left\{g\left(\pi^{-1}(\theta)\right) \mid(L, \pi) \text { supports } \xi\right\},
$$

where $\theta$ is any point in $S^{1}$ and $g\left(\pi^{-1}(\theta)\right)$ is the genus of the page $\pi^{-1}(\theta)$. The binding number of $\xi$ is defined to be

$$
\operatorname{bn}(\xi)=\min \left\{|L| \mid(L, \pi) \text { supports } \xi \text { and } \operatorname{sg}(\xi)=g\left(\pi^{-1}(\theta)\right)\right\},
$$

where $|L|$ denotes the number of components of $L$ (or, equivalently, the number of boundary components of any page of $(L, \pi))$. And the support norm of $\xi$ is defined to be

$$
\operatorname{sn}(\xi)=\min \left\{-\chi\left(\pi^{-1}(\theta)\right) \mid(L, \pi) \text { supports } \xi\right\},
$$

The first author was supported by an NSF Postdoctoral Fellowship and NSF Grant DMS-0635607. The second author was partially supported by NSF Grant DMS-0804820. 
where $\chi\left(\pi^{-1}(\theta)\right)$ denotes the Euler characteristic of any page $\pi^{-1}(\theta)$. It is a simple observation that $\operatorname{sn}(\xi) \geq-1$, with equality if and only if $\xi$ is the standard tight contact structure on $S^{3}$.

Since, for any surface $\Sigma$, we have the equality

$$
-\chi(\Sigma)=2 g(\Sigma)+|\partial \Sigma|-2,
$$

it is immediately clear that

$$
\operatorname{sn}(\xi) \leq 2 \operatorname{sg}(\xi)+\operatorname{bn}(\xi)-2 .
$$

Moreover, if the support norm of $\xi$ is achieved by an open book whose pages have genus $g>\operatorname{sg}(\xi)$ and whose binding has $m$ components, then $\operatorname{sn}(\xi)=2 g+m-2$, which is at least $2 \mathrm{sg}(\xi)+1$. The following lemma from [3] summarizes these bounds.

Lemma 1. For any contact structure $\xi$ on a closed, oriented 3-manifold,

$$
\min \{2 \operatorname{sg}(\xi)+\operatorname{bn}(\xi)-2,2 \operatorname{sg}(\xi)+1\} \leq \operatorname{sn}(\xi) \leq 2 \operatorname{sg}(\xi)+\operatorname{bn}(\xi)-2 .
$$

Thus, for contact structures with $\operatorname{bn}(\xi) \leq 3$, it follows that that $\operatorname{sn}(\xi)=$ $2 \operatorname{sg}(\xi)+\operatorname{bn}(\xi)-2$. Yet, the results in [3] do not resolve whether the upper bound on the support norm in Lemma 1 can ever be a strict inequality. Our main result is that this bound can indeed be a strict inequality; that is, the support genus and binding number do not, in general, wholly determine the support norm.

For the rest of this note, $\Sigma$ will denote the genus one surface with one boundary component. Let $\phi_{\mathbf{n}, m}$ be the diffeomorphism of $\Sigma$ given by

$$
\phi_{\mathbf{n}, m}=D_{\delta}^{m} \cdot D_{x} D_{y}^{-n_{1}} \cdots D_{x} D_{y}^{-n_{k}},
$$

where $x, y$ and $\delta$ are the curves pictured in Figure 1, and $\mathbf{n}=\left(n_{1}, \ldots, n_{k}\right)$ is a $k$-tuple of non-negative integers for which some $n_{i} \neq 0$. Let $\xi_{\mathbf{n}, m}$ denote the contact structure supported by the open book $\left(\Sigma, \phi_{\mathbf{n}, m}\right)$, and let $M_{\mathbf{n}, m}$ denote the 3-manifold with this open book decomposition.

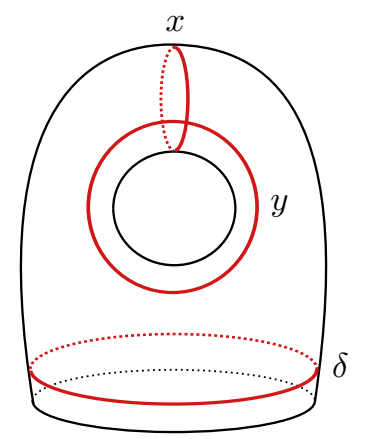

Figure 1. The surface $\Sigma$ and the curves $x, y$ and $\delta$. 
Theorem 2. For $m \leq 0$, the contact structure $\xi_{\mathbf{n}, m}$ satisfies

$$
\operatorname{sg}\left(\xi_{\mathbf{n}, m}\right)=0 .
$$

For any fixed tuple $\mathbf{n}$, there is a finite subset $E_{\mathbf{n}}$ of the integers such that

$$
\operatorname{bn}\left(\xi_{\mathbf{n}, m}\right)>3 \text { and } \operatorname{sn}\left(\xi_{\mathbf{n}, m}\right)=1
$$

for all $m \leq 0$ which are not in $E_{\mathbf{n}}$. In particular,

$$
\operatorname{sn}\left(\xi_{\mathbf{n}, m}\right)<2 \operatorname{sg}\left(\xi_{\mathbf{n}, m}\right)+\operatorname{bn}\left(\xi_{\mathbf{n}, m}\right)-2
$$

for all $m \leq 0$ which are not in $E_{\mathbf{n}}$.

In contrast, the support genus $\operatorname{sg}\left(\xi_{\mathbf{n}, m}\right)=1$ when $m>0$, [1]. Therefore, for $m>0, \operatorname{bn}\left(\xi_{\mathbf{n}, m}\right)=1$ and $\operatorname{sn}\left(\xi_{\mathbf{n}, m}\right)=1$, and, hence,

$$
\operatorname{sn}\left(\xi_{\mathbf{n}, m}\right)=2 \operatorname{sg}\left(\xi_{\mathbf{n}, m}\right)+\operatorname{bn}\left(\xi_{\mathbf{n}, m}\right)-2 .
$$

That is, the upper bound in Lemma 1 is achieved for $\xi=\xi_{\mathbf{n}, m}$ when $m>0$.

Proof of Theorem 2. One can easily see that, for $m \leq 0$, the open book $\left(\Sigma, \phi_{\mathbf{n}, m}\right)$ is not right-veering; therefore, for such $m$, the contact structure $\xi_{\mathbf{n}, m}$ is overtwisted [4] and $\xi_{\mathbf{n}, m}$ is supported by an open book with planar pages [2].

Observe that if a contact structure $\xi$ on $M$ is supported by an open book with planar pages and the binding number of $\xi$ is three or less, then $M$ must be a Seifert fibered space. More precisely, if the binding number of $\xi$ is two then $M$ is a lens space, and if the binding number is three then $M$ is a small Seifert fibered space. One can see this by drawing a surgery picture corresponding to the open book supporting $\xi$ which realizes the binding number.

It is well known that the diffeomorphism $\phi_{\mathbf{n}, 0}=D_{x} D_{y}^{-n_{1}} \cdots D_{x} D_{y}^{-n_{k}}$ is pseudo-Anosov (for instance, $\phi_{(1), 0}$ is the monodromy of the figure eight knot in $\left.S^{3}\right)$. Therefore, the binding of the open book given by $\left(\Sigma, \phi_{\mathbf{n}, 0}\right)$ is a hyperbolic knot, and the manifold $M_{\mathbf{n}, m}$ is obtained from $M_{\mathbf{n}, 0}$ via $-\frac{1}{m}$ surgery on this knot. Thurston's Dehn Surgery Theorem then implies that there is some finite subset $E_{\mathbf{n}}$ of the integers for which $M_{\mathbf{n}, m}$ is hyperbolic for all $m$ not in $E_{\mathbf{n}}\left[6\right.$. In particular, $M_{\mathbf{n}, m}$ is not a Seifert fibered space, save, perhaps, for some of the $m$ in the exceptional set $E_{\mathbf{n}}$. Hence, the binding number of $\xi_{\mathbf{n}, m}$ must be greater than three for all $m \leq 0$ which are not in $E_{\mathbf{n}}$.

We are left to check that the support norm of $\xi_{\mathbf{n}, m}$ is one when $m \leq 0$ and $M_{\mathbf{n}, m}$ is hyperbolic. If the support norm were not one, then it would be zero (the support norm must be non-negative since $\xi_{\mathbf{n}, m}$ is not the tight contact structure on $S^{3}$ ). But the only surface with boundary which has Euler characteristic zero is the annulus, and the only 3-manifolds with open book decompositions whose pages are annuli are lens spaces. 
It is natural to ask if the difference between $\operatorname{sn}(\xi)$ and $2 \operatorname{sg}(\xi)+b n(\xi)-2$ can be arbitrarily large. While we cannot answer this question we do note the following.

Theorem 3. For a fixed $\mathbf{n}$ the difference between $\operatorname{sn}\left(\xi_{\mathbf{n}, m}\right)$ and $2 \operatorname{sg}\left(\xi_{\mathbf{n}, m}\right)+$ $\operatorname{bn}\left(\xi_{\mathbf{n}, m}\right)-2$ is bounded independent of $m<0$.

Before we prove this theorem we estimate the binding numbers $\operatorname{bn}\left(\xi_{\mathbf{n}, m}\right)$ in some special cases.

Proposition 4. The binding number of $\xi_{(1),-1}$ satisfies $3 \leq \mathrm{bn}\left(\xi_{(1),-1}\right) \leq 9$. For each $m<-1$, the binding number of $\xi_{(1), m}$ satisfies $4 \leq \operatorname{bn}\left(\xi_{(1), m}\right) \leq 9$.

The manifold $M_{(1),-1}$ is the Brieskorn sphere $\Sigma(2,3,7)$. Since $\Sigma(2,3,7)$ is not a lens space, it does not admit an open book decomposition with planar pages and two or fewer binding components. Therefore, $\operatorname{bn}\left(\xi_{(1),-1}\right) \geq 3$. It is well-known that the only exceptional surgeries on the figure eight are integral surgeries [6]. Therefore, $E_{(1)}=\{-1,0,1\}$. So, from Theorem 2, we know that $\operatorname{bn}\left(\xi_{(1), m}\right)>3$ for all $m<-1$. To prove Proposition 4 , we construct an open book decomposition of $M_{(1), m}$ with planar pages and nine binding components and we show that it supports $\xi_{(1), m}$ for $m<0$.

Recall that overtwisted contact structures on a 3-manifold $M$ are isotopic if and only if they are homotopic as 2-plane fields. Moreover, the homotopy type of a 2-plane field $\xi$ is uniquely determined by its induced $S p i n^{c}$ structure $\mathbf{t}_{\xi}$ and its 3-dimensional invariant $d_{3}(\xi)$. Therefore, in order to show that the open book decomposition we construct actually supports $\xi_{(1), m}$ (for $m \leq$ 0 ), we need only prove that the contact structure it supports is overtwisted and has the same 3-dimensional invariant as $\xi_{(1), m}$ (their $\operatorname{Spin}^{c}$ structures automatically agree since $\left.H_{1}\left(M_{(1), m} ; \mathbb{Z}\right)=0\right)$. Below, we describe how to compute these invariants from supporting open book decompositions. For more details, see the exposition in [3].

Suppose that $\phi$ is a product of Dehn twists around homologically nontrivial curves $\gamma_{1}, \ldots, \gamma_{k}$ in some genus $g$ surface $S$ with $n$ boundary components. The open book $(S, i d)$ supports the unique tight contact structure on $\#^{2 g+n-1}\left(S^{1} \times S^{2}\right)$, and the $\gamma_{i}$ may be thought of as Legendrian curves in this contact manifold. The contact manifold $(M, \xi)$ supported by the open book $(S, \phi)$ bounds an achiral Lefschetz fibration $X$, which is constructed from $\sharp^{2 g+n-1}\left(S^{1} \times D^{3}\right)$ by attaching 2-handles along these Legendrian curves. Each 2-handle is attached with contact framing \pm 1 depending on whether the corresponding Dehn twist in $\phi$ is left- or right-handed, respectively. As long as $c_{1}\left(\mathbf{t}_{\xi}\right)$ is torsion in $H^{2}(M ; \mathbb{Z}), d_{3}(\xi)$ is an element of $\mathbb{Q}$ and may be computed according to the formula,

$$
d_{3}(\xi)=\frac{1}{4}\left(c^{2}(X)-2 \chi(X)-3 \sigma(X)\right)+q .
$$

Here, $q$ is the number of left-handed Dehn twists in the factorization $\phi$. The number $c^{2}(X)$ is the square of the class $c(X) \in H^{2}(X ; \mathbb{Z})$ which is Poincaré 
dual to

$$
\sum_{i=1}^{k} \operatorname{rot}\left(\gamma_{i}\right) C_{i} \in H_{2}(X, M ; \mathbb{Z}),
$$

where $C_{i}$ is the cocore of the 2 -handle attached along $\gamma_{i}$, and $\operatorname{rot}\left(\gamma_{i}\right)$ is the rotation number of $\gamma_{i}$. The class $c(X)$ restricts to $c_{1}\left(\mathbf{t}_{\xi}\right)$ in $H^{2}(M ; \mathbb{Z})$. Since we have assumed that $c_{1}\left(\mathbf{t}_{\xi}\right)$ is torsion, some multiple $k \cdot c(X)$ is sent to zero by the map $i^{*}: H^{2}(X ; \mathbb{Z}) \rightarrow H^{2}(M ; \mathbb{Z})$, and, hence, comes from a class $c_{r}(X)$ in $H^{2}(X, M ; \mathbb{Z})$, which can be squared. So, by $c^{2}(X)$, we mean $\frac{1}{k^{2}} c_{r}^{2}(X)$.

Lemma 5. For $m<0$, the 3-dimensional invariant $d_{3}\left(\xi_{(1), m}\right)=1 / 2$.

Proof of Lemma 5. Let $M_{(1), m}$ denote the result of $-\frac{1}{m}$ surgery on the figure eight knot. It is therefore a rational homology 3 -sphere, and the 3 dimensional invariant $d_{3}\left(\xi_{(1), m}\right)$ is a well-defined element of $\mathbb{Q}$. Observe that the Dehn twist $D_{\delta}$ is isotopic to the composition $\left(D_{x} D_{y}\right)^{6}$. As described above, the contact manifold supported by the open book $\left(\Sigma, \phi_{(1), m}\right)$ bounds an achiral Lefschetz fibration $X$, constructed from $\sharp^{2}\left(S^{1} \times D^{3}\right)$ by attaching $12|m|+2$ 2-handles corresponding to the Dehn twists in the factorization

$$
\phi_{(1), m}=\left(D_{x} D_{y}\right)^{6 m} \cdot D_{x} D_{y}^{-1} .
$$

From the discussion in [3. Section 6.1], it follows that $\operatorname{rot}(x)=\operatorname{rot}(y)=0$; hence, $c(X)=0$. Moreover, $\chi(X)=12|m|+1$ and $q=12|m|+1$. Therefore, the formula in (1) gives

$$
d_{3}\left(\xi_{(1), m}\right)=\frac{12|m|+1}{2}-\frac{3 \sigma(X)}{4} .
$$

The achiral Lefschetz fibration associated to the monodromy $\left(D_{x} D_{y}\right)^{2 m}$ gives a well-known Milnor fiber with the reverse orientation. Its signature is $8|m|$. One may easily check (via Kirby calculus or gluing formulas for the signature or computations of the degree of related Heegaard-Floer contact invariants) that $\sigma(X)=8|m|$. Thus $d_{3}\left(\xi_{(1), m)}\right)=1 / 2$.

Proposition 4 follows if we can find a planar open book with nine binding components which supports an overtwisted contact structure on $M_{(1), m}$ with $d_{3}=1 / 2$. The figure eight knot $K$ is pictured in Figure 2. $K$ can be embedded as a homologically non-trivial curve on the surface $S$ obtained by plumbing together two positive Hopf bands and two negative Hopf bands, as shown on the left in Figure 3 .

Topologically, $S$ is an embedded copy of the planar surface $P$ with five boundary components shown on the right in Figure 3. Moreover, $S$ is a page of the open book decomposition of $M_{(1), 0} \cong S^{3}$ given by $(P, \phi)$, where $\phi$ is the product of right-handed Dehn twists around the curves $\gamma_{1}$ and $\gamma_{3}$ and 


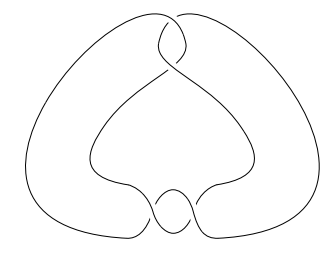

Figure 2. The figure eight, drawn here as a twist knot.

left-handed Dehn twists around the curves $\gamma_{2}$ and $\gamma_{4}$. The knot $K$ is the image, under this embedding, of the curve $r \subset P$.
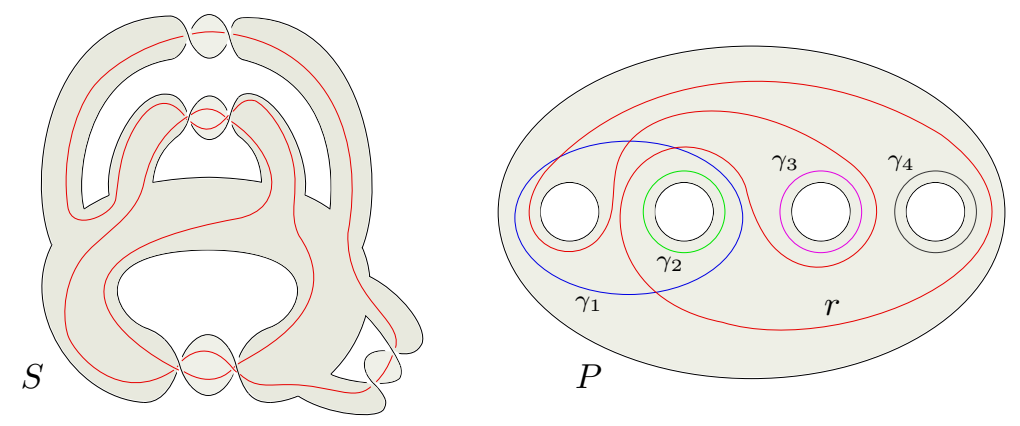

FiguRE 3. The figure eight knot, embedded on a planar surface with five boundary components.

Since the Seifert framing of $K$ agrees with the framing induced by $S$, $\left(P, D_{r}^{m} \cdot \phi\right)$ is an open book decomposition for $-\frac{1}{m}$ surgery on $K$. Let $\xi_{m}$ denote the contact structure on $M_{(1), m}$ which is supported by this open book. It is easy to check that the open book $\left(P, D_{r}^{m} \cdot \phi\right)$ is not right-veering for $m \leq 0$. (This can be seen by taking, for example, the horizontal arc connecting the right most boundary components of the surface on the right of Figure 3.) Therefore, the corresponding $\xi_{m}$ are overtwisted [4].

Lemma 6. For $m \leq 0$, the 3-dimensional invariant $d_{3}\left(\xi_{m}\right)=3 / 2$.

Proof of Lemma 6. Figure 4 shows another illustration of $P$, on the left; the four topmost horizontal segments are identified with the four bottommost horizontal segments to form 1-handles. As discussed above, we can think of these curves as knots in $\#^{4}\left(S^{1} \times S^{2}\right)=\partial\left(\sharp^{4}\left(S^{1} \times D^{3}\right)\right)$. The contact manifold supported by $\left(P, D_{r}^{m} \cdot \phi\right)$ bounds the achiral Lefschetz fibration $X$ obtained from $\sharp^{4}\left(S^{1} \times D^{3}\right)$ by attaching 2-handles along the curves $\gamma_{2}$ and $\gamma_{4}$ with framing +1 , along the curves $\gamma_{1}$ and $\gamma_{3}$ with framing -1 , and along $|m|$ parallel copies of $r$ (with respect to the blackboard framing) with framing +1 , as indicated on the right in Figure 4 .

Let $X, Y, Z$ and $W$ denote the 1-handles attached to $D^{4}$ to form $\sharp^{4}\left(S^{1} \times\right.$ $D^{3}$ ), as shown in Figure 4. Furthermore, let $S_{\gamma_{1}}, \ldots, S_{\gamma_{4}}$ and $S_{r_{1}}, \ldots, S_{r_{|m|}}$ 

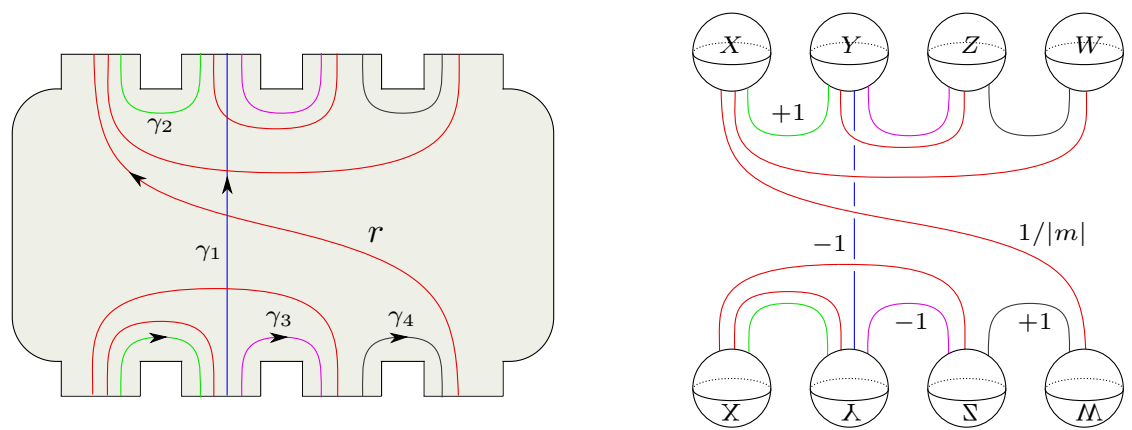

Figure 4. On the left, the surface $P$. On the right, a Kirby diagram for the achiral Lefschetz fibration corresponding to the open book $\left(P, D_{r}^{m} \cdot \phi\right)$. The label $1 /|m|$ indicates that we attach 2-handles along $|m|$ parallel copies of the curve $r$ with framing +1 .

denote the cores of the 2-handles attached to the curves $\gamma_{1}, \ldots, \gamma_{4}$ and the $|m|$ parallel copies $r_{1}, \ldots, r_{|m|}$ of $r$, and let $C_{\gamma_{1}}, \ldots, C_{\gamma_{4}}$ and $C_{r_{1}}, \ldots, C_{r_{|m|}}$ denote the cocores of these 2-handles. These cores form a basis for the group of 2-chains $C_{2}(X ; \mathbb{Z}) ; X, Y, Z$ and $W$ for a basis for the 1 -chains $C_{1}(X ; \mathbb{Z})$; and the boundary map $d_{2}: C_{2}(X ; \mathbb{Z}) \rightarrow C_{1}(X ; \mathbb{Z})$ sends

$$
\begin{aligned}
d_{2}\left(S_{\gamma_{1}}\right) & =Y, \\
d_{2}\left(S_{\gamma_{2}}\right) & =X-Y, \\
d_{2}\left(S_{\gamma_{3}}\right) & =Y-Z, \\
d_{2}\left(S_{\gamma_{4}}\right) & =Z-W, \\
d_{2}\left(S_{r_{i}}\right) & =-Z+Y+W .
\end{aligned}
$$

The homology $H_{2}(X ; \mathbb{Z})$ is therefore generated by $h_{1}, \ldots, h_{|m|}$, where

$$
h_{i}=S_{r_{i}}+S_{\gamma_{4}}-S_{\gamma_{1}} .
$$

By construction, $X$ may also be obtained from $D^{4}$ by attaching 2-handles along $|m|$ parallel copies of the figure eight with framing +1 , so the intersection matrix $Q_{X}$ is simply the $|m| \times|m|$ identity matrix with respect to the corresponding basis. Since the curves $r_{i}$ are parallel, it is clear that $h_{i} \cdot h_{j}=0$ for $i \neq j$. It follows that $h_{i} \cdot h_{i}=1$ for $i=1, \ldots,|m|$.

Recall that the class $c(X)$ is Poincaré dual to

$$
\sum_{i=1}^{4} \operatorname{rot}\left(\gamma_{i}\right) \cdot C_{\gamma_{i}}+\sum_{i=1}^{|m|} \operatorname{rot}\left(r_{i}\right) \cdot C_{r_{i}} .
$$

Via the discussion in [3, Section 3.1], we calculate that $\operatorname{rot}\left(\gamma_{4}\right)=\operatorname{rot}\left(\gamma_{2}\right)=$ $\operatorname{rot}\left(\gamma_{3}\right)=-1$ and $\operatorname{rot}\left(\gamma_{1}\right)=\operatorname{rot}\left(r_{i}\right)=0$. Therefore, $\left\langle c(X), h_{i}\right\rangle=-1$ for 
$i=1, \ldots,|m|$. So, thought of as a class in $H^{2}(X, \partial X ; \mathbb{Z}), c(X)$ is Poincaré dual to

$$
-h_{1}-\cdots-h_{|m|}
$$

Hence, $c^{2}(X)=|m|$. In addition, $\chi(X)=1+|m|, \sigma(X)=|m|$ and $q=$ $2+|m|$. From the formula in (11), we have

$$
d_{3}\left(\xi_{m}\right)=\frac{1}{4}(|m|-2(1+|m|)-3|m|)+2+|m|=3 / 2 .
$$

This completes the proof of Lemma 6.

Proof of Proposition 4 . Recall that

$$
d_{3}\left(\xi \# \xi^{\prime}\right)=d_{3}(\xi)+d_{3}\left(\xi^{\prime}\right)+1 / 2
$$

for any two contact structures $\xi$ and $\xi^{\prime}$. Let $m<0$. Since $d_{3}\left(\xi_{(1), m}\right)=1 / 2$, $d_{3}\left(\xi_{m}\right)=3 / 2$, and both $\xi_{(1), m}$ and $\xi_{m}$ are overtwisted, it follows that $\xi_{(1), m}$ is isotopic to $\xi_{m} \# \xi^{\prime}$, where $\xi^{\prime}$ is the unique (overtwisted) contact structure on $S^{3}$ with $d_{3}\left(\xi^{\prime}\right)=-3 / 2$. In [3], Ozbagci and the second author show that $\mathrm{bn}\left(\xi^{\prime}\right) \leq 5$. In particular, $\xi$ is supported by the open book $\left(P^{\prime}, D_{b}^{-1} D_{a}^{-1} \cdot \psi\right)$, where $a$ and $b$ are the curves on the surface $P^{\prime}$ shown in Figure 5 and $\psi$ is a composition of right-handed Dehn twists around the four unlabeled curves.

Then, the planar open book $\left(P \#_{b} P^{\prime}, D_{r}^{m} \cdot \phi \cdot D_{b}^{-1} D_{a}^{-1} \cdot \psi\right)$ with nine binding components supports $\xi_{(1), m} \simeq \xi_{m} \# \xi^{\prime}$, and the proof of Proposition 4 is complete. (Here, $\#_{b}$ denotes boundary connected sum.)

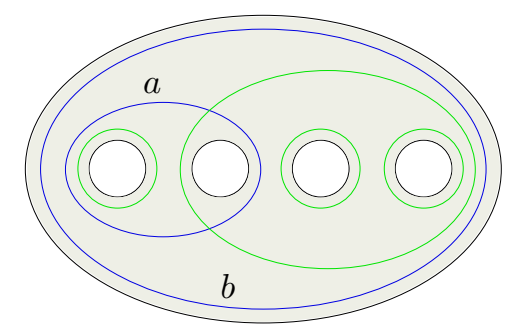

Figure 5. The surface $P^{\prime}$.

The table below summarizes what we know of the support genus, binding number and support norm for the contact structures $\xi_{(1), m}$.

Proof of Theorem 3. We first observe that $M_{\mathbf{n}, m}$ is a rational homology sphere. This can be seen by noticing that $M_{\mathbf{n}, 0}$ can be obtained as the 2-fold cover of $S^{3}$ branched over an alternating (non-split) link (in fact, closure of a 3-braid). Thus the determinant of the link is non-zero and hence the cardinality of the first homology of the cover is finite. Since $M_{\mathbf{n}, m}$ can be obtained from $M_{\mathbf{n}, 0}$ by $1 / m$ surgery on a null-homologous knot it has the same first homology. 


\begin{tabular}{|c||c|c|c|}
\cline { 2 - 4 } \multicolumn{1}{c||}{} & sg & bn & sn \\
\hline \hline$m>0$ & 1 & 1 & 1 \\
\hline$m=0$ & 0 & 1 & -1 \\
\hline$m=-1$ & 0 & {$[3,9]$} & 1 \\
\hline$m<-1$ & 0 & {$[4,9]$} & 1 \\
\hline
\end{tabular}

TABLE 1. Values of sg, bn and sn for $\xi_{(1), m}$.

Let $K$ be the binding of the open book $\left(\Sigma, \phi_{\mathbf{n}, 0}\right)$ in $M_{\mathbf{n}, 0}$. If we fix an overtwisted contact structure on $M_{\mathbf{n}, 0}$ we can find a Legendrian knot $L$ in the knot type $K$ with Thurston-Bennequin invariant 0 and overtwisted complement. In [5] it was shown that there is a planar open book $\left(\Sigma^{\prime}, \phi^{\prime}\right)$ for this overtwisted contact structure that contains $L$ on a page so that the page framing is 0 .

Notice that $M_{\mathbf{n}, m}$ can be obtained from $M_{\mathbf{n}, 0}$ by composing $\phi^{\prime}$ with a +1 Dehn twist along $m$ copies of $L$ on the page of the open book. Thus each $M_{\mathbf{n}, m}$ has an overtwisted contact structure supported by a planar open book with the same number of binding components. The number of $\operatorname{Spin}^{c}$ structure on $M_{\mathbf{n}, m}$ is finite and independent of $m$. We can get from the constructed overtwisted contact structure on $M_{\mathbf{n}, m}$ to an overtwisted contact structure realizing any $\operatorname{Spin}^{c}$ structure by a bounded number of Lutz twists along generators of $H_{1}\left(M_{\mathbf{n}, m}\right)$ all of which lie on a page of the open book. As shown in 2] we may positively stabilize the open book a bounded number of times and then compose its monodromy with extra Dehn twists to achieve these Lutz twists. The number of these stabilizations depends on the number of $\operatorname{Spin}^{c}$ structures on $M_{\mathbf{n}, m}$ and thus is independent of $m$. We now have planar open books realizing overtwisted contact structures representing all Spin $^{c}$ structures on $M_{\mathbf{n}, m}$ with the number of binding components bounded independent of $m$.

To get an open book representing any overtwisted contact structure on $M_{\mathbf{n}, m}$ we can take these and connect sum with overtwisted contact structures on $S^{3}$. In [3] it was shown that all overtwisted contact structures on $S^{3}$ have bn $\leq 6$. Thus we obtain a bound independent of $m$ on the binding number for all overtwisted contact structures on $M_{\mathbf{n}, m}$ and in particular on the $\xi_{\mathbf{n}, m}, m<0$.

Remark 7. One can also show, in a similar manner to the proof of Theorem [3, that the binding number of $\xi_{\mathbf{n}, m}$ is bounded by a constant depending only on the length of $\mathbf{n}$.

As noted in Theorem 2, $\operatorname{sg}\left(\xi_{\mathbf{n}, m}\right)=0$ for $m<0$ and $\operatorname{sn}\left(\xi_{\mathbf{n}, m}\right)=1$ for all but finitely many $m<0$; that is, these two quantities do not depend (much) on $\mathbf{n}$ or $m$. While we do know that the binding number of $\xi_{\mathbf{n}, m}$ is bounded 
independent of $m$ it could depend on (the length of) $\mathbf{n}$. This suggests the following interesting question, which we leave unanswered.

Question 8. Does there exist, for any positive integer n, a contact structure $\xi$ such that $2 \mathrm{sg}(\xi)+\operatorname{bn}(\xi)-2-\operatorname{sn}(\xi)=n$ ?

Note that $0 \leq 2 \operatorname{sg}\left(\xi_{(1), m}\right)+\operatorname{bn}\left(\xi_{(1), m}\right)-2-\operatorname{sn}\left(\xi_{(1), m}\right) \leq 6$ for all $m \in \mathbb{Z}$, but we currently cannot prove that this difference is larger that 1 . It would be very interesting to determine if there is an $m$ such that this difference is greater than 1. In general, computing $2 \operatorname{sg}\left(\xi_{\mathbf{n}, m}\right)+\operatorname{bn}\left(\xi_{\mathbf{n}, m}\right)-2-\operatorname{sn}\left(\xi_{\mathbf{n}, m}\right)$ could potentially provide a positive answer to this question.

Noticing that all our examples involve overtwisted contact structures we end with the following question.

Question 9. Is there a tight contact structure $\xi$ such that

$$
\operatorname{sn}(\xi)<2 \operatorname{sg}(\xi)+\operatorname{bn}(\xi)-2 \text { ? }
$$

\section{REFERENCES}

[1] J. A. Baldwin. Capping off open books and the Ozsváth-Szabó contact invariant. 2009, math.GT/0901.3797.

[2] J. B. Etnyre. Planar open book decompositions and contact structures. Int. Math. Res. Not., (79):4255-4267, 2004.

[3] J. B. Etnyre and B. Ozbagci. Invariants of contact structures from open books. Trans. Amer. Math. Soc., 360(6):3133-3151, 2008.

[4] K. Honda, W. Kazez, and G. Matić. Right-veering diffeomorphisms of a compact surface with boundary. Inv. Math., 169(2):427-449, 2007.

[5] Sinem Celik Onaran. Invariants of Legendrian knots from open book decompositions. 2009, arXiv:0905.2238

[6] W. Thurston. The geometry and topology of three-manifolds. Princeton, 1979.

Department of Mathematics, Princeton University

E-mail address: baldwinj@math.princeton.edu

$U R L:$ http://math.princeton.edu/ baldwinj

School of Mathematics, Georgia Institute of Technology

E-mail address: etnyre@math.gatech.edu

$U R L:$ http://math.gatech.edu/ etnyre 